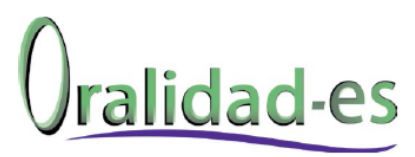

\title{
Instituciones de protección del patrimonio frente al conflicto armado y el papel de la oralidad como memoria cultural
}

\author{
Institutions for the protection of heritage against the armed \\ conflict and the role of orality as cultural memory
}

Ángel Ricardo Solano-Sanguino asolano49@unisalle.edu.co

Universidad de La Salle, Colombia

Solano-Sanguino, Á. R. (2020). Instituciones de Protección del patrimonio frente al conflicto armado y el papel de la oralidad como memoria cultural. Oralidad-es, 6, 1-15. https://revistaoralidad-es.com/index.php/ro-es/article/view/127 


\section{Resumen}

La protección del patrimonio cultural ha sido motivo de creación de derecho internacional, fomentado por organizaciones internacionales que han defendido a naciones vulneradas y afectadas directa o indirectamente por la destrucción, saqueo y tráfico de bienes culturales, así como por la prohibición o imposibilidad de transferencia oral de costumbres. Estos son considerados como daños irreparables para la humanidad, dado que, por una parte, el patrimonio cultural permite restablecer la identidad generando la creación de un vínculo con el pasado, el presente y el futuro y por la otra, la oralidad como memoria cultural es una expresión patrimonial compartida y su archivo corresponde a la memoria colectiva. Se utilizó una metodología de tipo cualitativa, documental y con orientación axiológica, con el fin de explicar las consecuencias del expolio como por ejemplo el nazi, el cual generó además de la pérdida aproximada de 50 millones de vidas, daños al patrimonio cultural material e inmaterial de toda Europa. Se halló que los países en las invasiones son motivados por intereses oscuros como erradicar la presencia histórica, bienes tangibles e intangibles, así como la oralidad y el futuro de la comunidad atacada.

\section{Palabras Clave}

Protección; patrimonio cultural; transmisión oral; expolio; conflicto armado; memoria cultural.

\section{Abstract}

The protection of cultural heritage has been a motive for the creation of international law, promoted by some international organizations that have defended nations that have been violated and affected directly or indirectly by the destruction, looting and trafficking of cultural property, as well as by the prohibition or impossibility of oral transfers of customs. These are considered to be irreparable damage to humanity, given that cultural heritage makes it possible to restore identity by creating a link with the past, present, and future. A qualitative, documentary and axiologically oriented methodology was used in order to explain the Nazi plundering, which generated not only the loss of approximately 50 million lives, but also damage to tangible and intangible cultural heritage throughout Europe. It was found that the countries in the invasions are motivated by obscure interests such as eradicating the historical presence and the future of the attacked community.

\section{Keywords}

Protection; cultural heritage; oral transmission; plunder; armed conflict; cultural memory. 


\section{Introducción}

Para comenzar, se explora el concepto patrimonio cultural por medio de una breve revisión bibliográfica, ya que de esta manera se pudo realizar un acercamiento a dicho concepto, y en ese sentido, identificar qué engloba y qué alcance posee el patrimonio cultural y de esta forma tomar conciencia respecto a la importancia de la transmisión cultural del patrimonio al interior de las comunidades.

En relación al patrimonio cultural es definido por The United Nations Educational, Scientific and Cultural Organization (UNESCO, 1972), Instituto Latinoamericano de Museos y Parques (ILAM, 2020), Comunidad Andina (CAN, s.f.a), Ministerio de Cultura de Colombia (MinCultura, 2015) y Pinassi (2018), como una construcción social que abarca un conjunto de bienes tangibles e intangibles producto de la creatividad humana, que se transmiten, heredan y modifican de generaciones pasadas, se viven en el presente e inciden en poblaciones futuras. En esta definición, llama la atención el énfasis y el reconocimiento que se le da a los bienes intangibles, representados en valores simbólicos, espirituales, estéticos y tecnológicos.

En este contexto, la oralidad cumple un papel fundamental por su variedad de formas de expresión distantes y distintas que reflejan las psicodinámicas socioculturales del individuo y las comunidades. Estas particularidades expresivas se transmiten y encarnan costumbres, tradiciones, conocimientos y, en definitiva, crean un acervo cultural; por tanto, la oralidad es una expresión patrimonial compartida, su existencia se multiplica al tener en cuenta su conformación en el tiempo y en el espacio, su intencionalidad y sus efectos; por tanto, las diferentes generaciones juegan un rol importante en la conformación de una cultura común (Espinosa, et al., 2018). En consecuencia, se resalta la importancia de la oralidad de las comunidades como un componente fundamental del acervo cultural, cuya conservación es fuente de información, medio de comunicación y patrimonio inmaterial de la humanidad.

De forma parecida, Piarpusan, et al. (2020) señala que, la oralidad se puede denominar como una riqueza cultural e inmaterial que pasa a la memoria y esto lleva a la construcción de los procesos y estructuras que permiten que el conocimiento se siga transmitiendo. Por tal motivo, las prácticas sociales generadas en el pasado, transmitidas de forma oral y que se viven en el presente deben ser protegidas y respetadas, puesto que son un auténtico recurso para mantener viva la herencia cultural en la memoria de las nuevas generaciones (Rodríguez, 2004, citado en Espinosa-Sosa, Espinosa-Sosa y Abad-Saínz, 2018, p.5).

Por otro lado, la UNESCO (1972), profundiza en el patrimonio cultural de cada Estado, específicamente en los bienes tangibles, asegurando que estos están conformados por monumentos, grupos de edificios o sitios; caracterizados por poseer valor universal en cuanto a factores históricos, artísticos, naturales o científicos. Por ende, se reconoce que no solo las creencias, los símbolos o los principios obtienen una significancia cultural, sino también el patrimonio cultural material, dado que estos son fundamentales para las comunidades, pues en su mayoría tienen un alto grado de valor simbólico que los une con sus antepasados y en general con su cultura.

En ese sentido, el patrimonio cultural material no solo lo conforma lo tangible: libros, monumentos, ciudades, edificios, obras de arte, joyas, etc., sino que estos también poseen un valor simbólico, es decir, lo material se vuelve simbólico o inmaterial. (Gertz,1997). En la década del 80 en pleno momento de auge del giro lingüístico, la significación cultural es redefinida en la carta sobre el valor cultural de Burra de 1979 (y sus posteriores revisiones) a lo relacionado con aspectos intangibles como cualidades simbólicas y recuerdos; a ello se le suma que el concepto de 
significación cultural es concebido como sinónimo de valor cultural. La oralidad entonces adquiere un significado cultural como una construcción conceptual compleja que vincula las etapas de la vida histórica de un bien patrimonial que permite comprender su razón de ser en el tiempo, detectar lo que es importante en la vida de los mismos y explicarlo como producto cultural. (Manzzini, 2009). Asimismo, lo inmaterial se puede volver material; en general, el valor simbólico que adquieren los objetos pueden generar conductas, actitudes e intereses dentro de una comunidad y estos a su vez generan un pasado, un presente y un futuro en común. Por ejemplo: "una partitura musical como objeto tiene tanto significado como el sonido que de ella pueda surgir a través de la interpretación de un músico” (Valencillos, 2013, citado en Paz, Cedeño \& Macías, 2016, p.123). En efecto, depende de la comunidad la importancia que le otorgue a este tipo de bien cultural.

Se puede entender entonces, el patrimonio cultural de una manera muy amplia, a pesar de que, a primera vista, parece fácil definir el concepto de patrimonio cultural, esto no es así, ya que puede abarcar cualquier objeto realizado dentro de un territorio, siendo esto uno de los mayores problemas generados al momento de la repatriación de dichos objetos por parte de un Estado a otro (Verón, 2018). Por consiguiente, queda a disposición de las autoridades competentes de cada Estado definir cuáles son los bienes considerados como monumentos, patrimonio cultural bien sea por su importancia arqueológica o histórica (MinCultura, 2015, p.2).

Pese a lo anterior, aún es complejo lograr una comprensión general de lo que es el Patrimonio Cultural Inmaterial y sus relaciones con los conceptos de oralidades y comunidades. De ahí que esta investigación, tome como referente las consecuencias del expolio nazi, el cual generó daños en el patrimonio cultural material e inmaterial de toda Europa.

\section{Metodología}

En primer lugar, la metodología que se utilizó en este trabajo es de tipo cualitativa en el sentido planteado por Cagliani (2015), en cuanto a que, "busca adquirir información en profundidad para poder comprender el comportamiento humano y las razones que gobiernan tal comportamiento" (p.1).

En segundo lugar, se hizo énfasis en la investigación documental, ya que, según Socorro, Reyes \& Trujillo (2019), aseguró que los datos recopilados provienen de fuentes como "libros, artículos, videos, revistas, publicaciones periódicas, [entre otras]” (p.2). Estas fuentes permiten relacionar, contrastar y se vincula en la investigación propuesta.

En tercer lugar, el presente trabajo de investigación posee orientación axiológica debido a que basados la Universidad Nacional Autónoma de México (UNAM, s.f.), explicó que, la axiología o «teoría de los valores» "reflexiona sobre la naturaleza y pertinencia de los mismos, sin reducirse a juicios de valor o preferencias estimativas del todo subjetivas" (p.2). También, el mismo autor afirma que esos valores pueden ser normalizados, convirtiéndose en estándares de comportamiento que puede establecer lo que se puede y no se puede hacer, lo bueno y lo malo.

Por último, se presentan resultados de la investigación desde un razonamiento deductivo en los términos planteados por el Monterey Institute for Technology and Education (2011), cuando "usamos hechos conocidos para llegar a conclusiones lógicas que sabemos son verdaderas. (Deducimos un hecho al unir otros factores.) Esto es distinto que el razonamiento inductivo, que generaliza y conjetura basado en observaciones en lugar de lógica" (p.1). 
Expolio de arte. Siguiendo a Scotland Yard y a las investigaciones del Federal Bureau of Investigation (citado en Mora, 2018), apuntan que el robo de arte se encuentra en el segundo puesto de los delitos de tráfico más lucrativos, ubicándose solo detrás del negocio de las drogas. Igualmente, aseveran que los involucrados en los delitos de expolio de arte en su mayoría están relacionados con otros delitos como tráfico de armas y drogas. Estos pueden llegar a estar compuestos por anticuarios, marchantes, pasando por delincuentes comunes, bandas criminales y hasta narcotraficantes, terroristas o incluso guerrillas.

\section{Sin embargo, se puede señalar que de Scotland} Yard y del Federal Buró han olvidado la relevante incidencia, no solo por parte de privados y de grupos al margen de la ley, sino que históricamente también han existido vulnerabilidades por parte de Estados en busca de recursos y en efecto, una afectación cultural. Cabe añadir que, el daño generado para las comunidades victimas es inadmisible, dado que el robo o destrucción de un bien tiene un alto costo simbólico y subjetivo, pero de gran relevancia para la cultura pues puede provocar una desconexión con el pasado y sus procesos de construcción de memoria desde la oralidad.

\section{Problemática cultural que} amenaza la memoria. La Asociación para la Investigación de Crímenes contra el Arte (ARCA, s.f.), afirma que los delitos cometidos con mayor frecuencia son los saqueos en los museos y los robos ocurridos durante los conflictos internos y guerras entre Estados. De ahí, la gran importancia que representa la demanda y oferta del arte, dado que este es uno de los motores que más ha incentivado el robo. Según el $21^{\circ}$ Informe Anual del Mercado del Arte mundial (citado por Cision, 2019), el mercado del arte se encuentra en gran crecimiento, está entrando en una nueva era y en el2018 las ventas en las subastas de Fine Art alcanzaron 15,48 mil millones de dólares. Es decir, se vendieron 538.000 obras. Los Estados Unidos poseen una variación porcentual de $+18 \%$ y el Reino Unido de $+12 \%$ con respecto al año anterior. Además, lideran el crecimiento de Occidente. Estados Unidos es la primera potencia mundial con 5,8 mil millones de dólares (es decir, el 37,9\% de las ventas mundiales) y China se coloca justo detrás, con 4,5 mil millones de dólares.

Basados en Cision (2019) y Verón (2018), la anterior panorámica, da cuenta del Mercado del Arte con unas características importantes, tales como: histórico, eficiente, mundial y con facilidad para resistir a las crisis económicas. En ese sentido, Verón (2018), ahonda en el tema, aseverando que en tiempos de crisis los inversores en su mayoría confían en valores con poca incertidumbre como lo son obras de arte o metales preciosos. Lo anterior, se puede observar el aumento del 30\% de los precios mundiales del oro en el actual contexto pandemico de la COVID-19, debido a su estatus de factor refugio (Portafolio, 2020).

\section{Expolio y destrucción de patrimonio cultural en conflicto}

armado. Un fenómeno inédito es el crecimiento del tráfico de arte expoliado y la destrucción de patrimonio cultural en los conflictos armados ha sido uno de los enemigos relevantes del patrimonio cultural, en este caso Vinson (2003), Torrecuadrada (2013) y Verón (2018), concuerdan que el factor histórico en las diferentes civilizaciones ha provocado preocupantes consecuencias de diversa índole, las cuales pueden ir desde el interés de conquistar nuevos territorios, daños colaterales hasta obtención de botines de guerra, teniendo en cuenta que en la práctica pueden confluir entre ellos. 
Ahora bien, Vinson (2003) y Torrecuadrada (2013) mencionan la limpieza cultural como un factor causante de destrucción de patrimonio cultural en conflicto armado, hecho que llama la atención, básicamente por su destrucción intencionada la cual tiende a ser más cruel que las anteriores, dado que tienen el propósito de borrar la memoria histórica haciendo desaparecer los bienes tangibles e intangibles de un grupo étnico o religioso y, en consecuencia, sus raíces y su futuro, este caso se puede clasificar como un instrumento evidente de etnocidio.

\section{Protección del patrimonio cultural en conflicto armado. SLa} repatriación de patrimonio cultural en tiempos de guerra no es novedosa, es tan antigua como la historia de la humanidad (Torrecuadrada, 2013); sin embargo, es un precedente que se debe tener en cuenta y, lo que sí es de franca actualidad es el establecimiento de mecanismos para su restitución o recuperación y entrega a los legítimos propietarios. Además, asegura que no se puede dejar de rememorar que la evolución del Derecho Internacional ha modificado la crítica jurídica sobre la incautación de patrimonio cultural en la guerra; es decir, en el Derecho Internacional clásico, cuando el principio de no intervención aún no existía, la destrucción o expolio de patrimonio cultural no vulneraba ninguna norma, básicamente era una práctica lícita.

En otras palabras, en un inicio los botines de guerra, estaban permitidos y cabe destacar que eran los premios que obtendrían los vencedores. En consecuencia, según Torrecuadrada (2013), "no es hasta entrado el siglo XIX cuando comienza a adquirir conciencia de la necesidad de evitar la desaparición de los objetos artísticos y culturales durante los enfrentamientos bélicos" (p.45).

Según Torrecuadrada (2013), concluida la Segunda Guerra Mundial, se crea la necesidad de una norma general que abarque la posible restitución de los bienes culturales incautados en tiempos de conflicto armado, hasta la fecha sólo existen algunas normas creadas por los diferentes tratados de paz. Estos no involucran soluciones de tipo más generales, sino que se limitan a circunstancias particulares, inclusive de esto se utilizó en materia jurídica la sucesión de Estados provocada por el nuevo trazado de algunas fronteras y, con este, el cambio del Estado en cuyos territorios se encontraban los edificios, castillos, etc., que habían sobrevivido a la contienda, así como los bienes artísticos, culturales o religiosos que contenían en su interior.

En el estudio realizado a algunos tratados de paz que reconocían la importancia de los bienes culturales, Torrecuadrada (2013), como lo fueron: el Tratado de Paz firmado en París el 30 de mayo de 1814, entre Francia, Austria, Rusia, Gran Bretaña, Prusia, Portugal y Suecia; los Tratados de Paz concluidos como consecuencia de la Primera Guerra Mundial, se encontró que estos refieren de modo diverso a los bienes desplazados durante la contienda. Así, por ejemplo, el Tratado de Versalles; Tratado de Saint Germain en Laye en relación con Austria y de Trianon sobre Hungría; Tratado de Neuilly (Tratado de Paz con Bulgaria); el Tratado de Riga, celebrado entre Ucrania y Rusia, de una parte y Polonia, de otra; siendo el texto más completo y complejo en lo que a la restitución se refiere de los celebrados hasta ese momento.

Enfocados en la sucesión de Estados, Torrecuadrada (2013), afirma que estos fueron: el Decreto de 21 de junio de 1945, sobre la confiscación y el reparto acelerado de tierras agrícolas de los nacionales alemanes y húngaros y de los traidores $\mathrm{y}$ enemigos del pueblo checo y eslovaco, conocidos como los “Decretos Benes”. La Declaración Conjunta que adoptaron las Potencias Aliadas el 5 de enero de 1943, además de incorporar su compromiso de colaboración para evitar los efectos del expolio, negó el efecto de las confiscaciones directas e indirectas en los territorios ocupados. 
Por su parte, la Conferencia de Bretton Woods, incorporó una Resolución (la Sexta) en la que, se recomendó a los Estados representados en este evento que insistían en los países neutrales, evitar cualquier transferencia de activos, moneda, oro, objetos de arte, etc., provenientes de los territorios ocupados, con independencia de su naturaleza pública o privada. Finalmente, los acuerdos de paz de la Segunda Guerra Mundial, en los que participaron las potencias vencedoras, también incorporaron disposiciones específicas a la restitución concreta y puntual de bienes.

\section{Defensores del patrimonio cultural.}

\section{Organizaciones gubernamentales.}

A continuación, se presentan algunas de las principales organizaciones gubernamentales que se han tomado la tarea de salvaguardar, proteger y recuperar patrimonio cultural, en especial aquellos que han sido más vulnerados.

The United Nations Educational, Scientific and Cultural Organization (UNESCO)

La Organización de las Naciones Unidas para la Educación, la Ciencia y la Cultura (UNESCO) por sus siglas en inglés, es un organismo especializado del Sistema de las Naciones Unidas (ONU). Su objetivo está basado en el respeto de valores comunes con el fin de generar una cooperación entre civilizaciones, pueblos y culturas que contribuyan a preservación de la paz y a la seguridad mundial (UNESCO, citado en Gobierno de México, s.f., p.1). La UNESCO (1972) en la convención sobre la protección del patrimonio mundial, cultural y natural, estipula que "la Organización ayudará a la conservación, al progreso y a la difusión del saber, velando por la conservación y la protección del patrimonio universal” (p.2).

En este punto, la UNESCO reconoce al patrimonio cultural y al patrimonio natural en peligro de destrucción, no sólo por las causas tradicionales de deterioro sino también por la evolución de la vida social y económica que las agrava con fenómenos de alteración o de destrucción aún más temibles, por lo tanto, el tema de la protección del patrimonio cultural, busca "velar por la conservación y protección universal de libros, obras de arte y monumentos de interés histórico o científico" (Lizarauzu, 2016, p.8).

International Institute for the Unification of Private Law (UNIDROIT). El Instituto Internacional para la Unificación del Derecho Privado (UNIDROIT) en sus siglas en inglés, es una "organización intergubernamental independiente creada en 1995 en el Convenio de UNIDROIT como complemento de la Convención de la UNESCO de 1970. UNIDROIT se centra en un manejo uniforme en los procesos de restitución de bienes culturales expoliados y traficados ilícitamente, facilitando el debido proceso de las demandas directamente en los tribunales nacionales. Específicamente, este convenio busca reducir el tráfico ilícito de todos los bienes culturales y declara que todo bien cultural robado debe ser restituido (UNIDROIT, 2014).

International Criminal Police Organization (INTERPOL). La Organización International de Policía Criminal (INTERPOL) por sus siglas en inglés, es una organización intergubernamental que cuenta con 194 países miembros; ayudan a la policía de estos países a colaborar entre sí para hacer del mundo un lugar más seguro (INTERPOL, s.f.a.). Con referencia al expolio de bienes culturales, la INTERPOL considera la protección del patrimonio como un problema imperativo de seguridad. En ese sentido, establece que los delitos contra el patrimonio cultural no pueden ser considerados simplemente como robos de objetos, sino que puede estar dirigida por la persecución y erradicación de las raíces culturales de comunidades enteras (INTERPOL, s.f.b.). 
Comunidad Andina (CAN). La Comunidad Andina es una organización internacional la cual está conformada por diversos órganos e instituciones que integran el Sistema Andino de Integración (SAI), su objetivo general es lograr un desarrollo integral fundamentado en la cooperación sudamericana (CAN, s.f.b.). Asimismo, CAN hace énfasis en la protección de bienes culturales, para esto creó una norma comunitaria basada en el fomento de la protección y conservación del patrimonio cultural. Se trata de la Decisión 588, aprobada en julio de 2004 que tiene como fin crear y ejecutar planes conjuntos que eviten la extracción e ingreso de patrimonio cultural entre los países miembros y terceros países, por medio de la conservación, protección, vigilancia y repatriación de estos bienes (CAN, s.f.a, p.1).

Entre las acciones que la Comunidad Andina se propone desarrollar un proyecto transfronterizo entre Colombia, Bolivia y Ecuador para la protección y salvaguardia del patrimonio cultural intangible como los conocimientos tradicionales relacionados con la naturaleza amazónica (CAN, s.f.a.)

\section{Organizaciones no gubernamentales.}

\section{International Council of Museums}

(ICOM). El Consejo Internacional de Museos (ICOM) por sus siglas en inglés, es una organización no gubernamental conformada por museos y profesionales del tema en todo el mundo, cuyo objetivo es investigar, perpetuar, perennizar y transmitir a las sociedades el patrimonio cultural y natural mundial, presente y futuro, tangible e intangible. En relación con el patrimonio cultural, hacen recomendaciones, promueven la creación de capacidades y avanzan en el conocimiento de dicho tema. ICOM se puede reconocer como la voz de los profesionales de museos en el escenario internacional, aumentan la conciencia cultural pública a través de redes mundiales y programas de cooperación (ICOM, s.f.).
Monuments Men Foundation for the Preservation of Art. Es una fundación que honra el legado de los hombres y mujeres que sirvieron en la sección Monuments Men Foundation for the Preservation, en adelante (MFAA).

Estos intentan preservar la historia de "Monuments Men" y aprender de ella para asegurar un mejor mañana. Su misión es educar al público sobre estos heroicos veteranos, facilitando la devolución de innumerables obras de arte y bienes culturales a sus legítimos propietarios, y trabajando para establecer principios sobre el respeto de los bienes culturales de los demás (Monuments Men Foundation for the Preservation of Art, s.f).

Recopilando lo dicho, se puede agregar que la conciencia que conlleva la pérdida y las consecuencias que ha generado el detrimento del patrimonio cultural a lo largo de los años, ha permitido que algunos Estados y organizaciones gubernamentales y no gubernamentales, hayan intervenido para evitar tanto la destrucción como el robo de estos. Por lo tanto, dichos actores, se enfocan en ayudar a reconstruir comunidades desmembradas, a restablecer su identidad, a crear un vínculo con su pasado, su presente y su futuro (UNESCO, s.f.a). Sin embargo, también existen algunos Estados que han hecho caso omiso tanto a tratados como a acuerdos, y no estan dispuestos a regresar ningún tipo de estos objetos (Semana, 2013). De hecho, algunos han seguido saqueando ciudades como ocurrió en Bagdad por tropas de Estados Unidos (Albert, 2015). 


\section{Expolio nazi}

Para analizar la protección del patrimonio cultural en conflicto armado, se expone el caso del robo y destrucción de bienes culturales llevados a cabo por la Alemania nazi en la Segunda Guerra Mundial. No sin antes, resaltar un argumento basado en Torrecuadrada (2013), ya que refleja que:

(...) ni el Derecho Internacional ni la Sociedad de Naciones fueron capaces de evitar la Segunda Guerra Mundial, que asoló con virulencia principalmente el continente europeo. [Como se va a presentar más adelante], esta contienda plantea una complejidad incomparable de problemas derivados de la destrucción y el saqueo, seguido del desplazamiento de bienes artísticos (p.82).

Conforme el frente de los alemanes avanzaba y se expandía por toda Europa estos fueron acumulando en depósitos seguros colecciones de bienes artísticos robados por todo el antiguo continente, y poniéndolos a salvo de posibles bombardeos, emplazados generalmente en búnkeres bajo tierra o en minas de sal (Cardona, 2013). De manera simultánea, las fuerzas de los aliados creaban MFAA, la cual nace en septiembre de 1943, tras el desembarco en Sicilia (Edsel, citado en Martorell-Linares, 2014).

Los diferentes hombres y mujeres, quienes integraron este programa eran provenientes de trece naciones, la mayoría se ofreció voluntariamente para el servicio en la recién creada sección MFAA. Cabe destacar que, estas personas tenían experiencia como directores de museos, curadores, académicos de arte, educadores, artistas, arquitectos y archiveros, algunos ya habían pasado la edad para estar en la guerra sin embargo fueron hasta ella para cumplir su misión (Martorell-Linares, 2014).
Su trabajo era simple, a lo largo de la guerra y la inmediata posguerra cumplirían una doble misión: proteger el patrimonio artístico europeo en el frente de guerra y, conforme los aliados iban ocupando nuevos territorios lograr recuperar las obras de arte expoliadas por los nazis para restituirlas a sus legítimos propietarios (Edsel, citado en Martorell-Linares, 2014).

Es decir, las MFAA no solo trataron de preservar monumentos en el frente conforme este iba avanzando, sino que también en la retaguardia para inventariar las piezas destruidas durante la guerra y, sobre todo aquellas que habían sido rapiñadas por los alemanes de las colecciones públicas o privadas y de edificios religiosos. Esta segunda función de las MFAA fue esencial para reconstruir el panorama artístico europeo en la posguerra, así lo expresa Edsel citado por Martorell-Linares (2014).

Cabe resaltar que, las MFAA recuperaron cerca de 1.500 de estos depósitos que contenían obras de art, tanto robadas como evacuadas de los museos por los alemanes. Por mencionar algunos de esos depósitos fueron, las minas de sal de Merkers, en la cual se hallaron aproximadamente 400 obras de arte y oro de propiedad del reischbank; el Castillo de Neuschwanstein, este se caracterizó por poseer en su mayoría bienes artísticos pertenecientes a Francia, los cuales incluyeron alrededor de 6.000 obras artísticas como joyas, oro, muebles, entre otros; las minas de sal de Altaussee en Austria, en esta se encontraron más de 6.500 obras de arte (Cardona, 2013).

De hecho, desde mediados del pasado siglo, el expolio de obras de arte y de otro tipo de bienes, constituye una de las líneas de investigación más fructíferas, debido a la gran cantidad de arte que ha sido despojado de sus verdaderos dueños al paso de los siglos. La caída del muro de Berlín, el final de la guerra fría, la descomposición de la URSS y la reunificación de Alemania, hicieron que retornaran a un primer plano en la 
actualidad diversos problemas, los cuales fueron cerrados en falso en la posguerra, entre ellos, la devolución de los bienes expoliados por los nazis a sus legítimos dueños, así lo afirma Martore11-Linares (2014).

En consecuencia, la Comisión para el Arte Incautado en Europa calcula que, durante la Segunda Guerra Mundial, la Alemania Nazi despojó a la comunidad judía europea alrededor de 600.000 bienes tangibles. Sin embargo, fue hasta los noventa que el proceso de restitución comenzó, básicamente por la presión ejercida por investigadores e historiadores (Semana, 2013).

Por medio de este escrito, se buscó explicar la causa de la destrucción y saqueo del patrimonio cultural europeo y judío. Se partió de la tesis de que los Estados responden a intereses materiales. Ahora bien, para esto se exploró algunas fuentes bibliográficas con el motivo de hacer un acercamiento al concepto de patrimonio cultural, expolio de arte y consecuencias culturales e identitárias. Asimismo, se describieron las acciones de los países y de organizaciones internacionales sobre la repatriación cultural y, por último, se buscó reconocer la importancia del patrimonio cultural para la construcción de la identidad con el caso del expolio nazi.

\section{Discusión}

Se halló que el objetivo de las organizaciones internacionales a pesar de su componente (racional-legal) ha sido perturbado en su eficiencia, dado a la cantidad de patologías que poseen. Dado que estas organizaciones son permeadas por los intereses de algunos Estados, desvían sus principales objetivos, limitándose al cumplimento de las normas. Por ende, el poco poder que puede llegar a tener una organización es un poder simbólico basado en la legitimidad, en lo que son, en lo que significan y no en lo que realmente hacen. En consecuencia, los Estados viendo la incapacidad de poder coercitivo de las organizaciones hacen caso omiso de toda la legislación y de las normas creadas por estas.

Entonces, solo de buenas intenciones no se puede lograr la repatriación tal como ocurrió con el presidente Macron, el cual tomó la decisión de devolver un objeto robado por Francia en la época colonial. Sin embargo, esta decisión no fue ejecutada, dado que no se puede tomar sin la aprobación del parlamento francés, básicamente el presidente no puede devolver el patrimonio cultural de la nación, incluso si lo adquirieron por medio de la coerción o el fraude. Es decir, para que se pueda dar la repatriación en estos Estados blindados jurídicamente, es fundamental realizar cambios estructurales lo cual es poco probable, pues estos establecen algunos obstáculos jurídicos como que la nación repatriada debe tener la capacidad de preservarlos (Semana, 2019, p.4).

Este último argumento, es bastante utilizado por los países intervencionistas que han saqueado grandes culturas a lo largo de los años, como expresa Medina (citado en Semana, 2019), que salvo algunas excepciones, tanto como América Latina y África deberían agradecer el cuidado que Europa le ha dado al legado de la humanidad, ya que hubieran podido desaparecer por la inestabilidades políticas, sociales, económicas y hasta naturales, y por el contrario, deberían ser exhibidos en los grandes museos.

Ahora bien, se debe reconocer que para "comprobar la posesión de una obra de arte cuando ha pasado tantos años, no es nada fácil. Se necesitan pruebas jurídicas, testamentos, recibos de compra, fotografías, inscripciones o sellos en el reverso del cuadro" (Feliciano, citado en Semana, 2013, p.4).

Igualmente, se encontró que el patrimonio cultural lo abarca tanto el patrimonio material como inmaterial, los cuales están conectados y poseen un papel de gran relevancia para la sociedad, ya que permite la construcción de la 
identidad cultural de un pueblo. Esto se debe básicamente porque la identidad "viene definida históricamente a través de múltiples aspectos en los que se plasma su cultura, como la lengua, [...] las relaciones sociales, ritos y ceremonias propias, o los comportamientos colectivos" (Gonzales, citado en Molano, 2007, p.73). Sobre todo, porque la identidad brinda sentido de pertenencia a un grupo social o comunidad, pues crea lazos que los conectan con su pretérito, generando su práctica y respeto continuo.

Como resultado, se encontró la relevancia que poseen las culturas independientemente del lugar de origen, de las prácticas o principios en que estas se basen, pues queda claro que son fundamentales para la construcción de la identidad y de un futuro común, por ende, se exhorta el respeto de la diversidad cultural como un bien de la humanidad que debe ser respetado.

De forma parecida, se halló que las tradiciones orales se convierten en una forma de patrimonio cultural inmaterial y tradicional de la comunidad en que se manifiesta, puesto que permite la transmisión de tradiciones, conocimientos, patrones de convivencia social y expresiones orales, las cuales se traducen en la conservación de la memoria colectiva (Espinosa, et al., 2018).

Las diferentes expresiones orales eran prácticas de ritualización social, por tanto, su prestigio cultural e intelectual estuvo dominado por prácticas escritas y gráficas (libros, obras de arte, etc.). De ahí, la necesidad de recuperar su valor retórico, su estrecha relación con la construcción colectiva de la sociedad, por ahora conminado a museos, bibliotecas y universidades.

En consecuencia, se puede afirmar que es clara la necesidad que tienen las naciones en la protección de su patrimonio cultural material e inmaterial. Sin embargo, prima la búsqueda de los intereses materiales por parte de los Estados, debido a que es una de las razones para que decidan robar o destruir el patrimonio cultural de estas comunidades y territorios.
En cuanto al expolio Nazi, se pudo detectar que Adolf Hitler entendió el significado de la cultura bajo su propio interés y buscó afectar por completo la memoria cultural judía y toda aquella que no fuera acorde a su pensamiento, es decir, uno de sus propósitos fundamentales, además de su sed por conquistar territorios y su engrandecimiento fue la de borrar la memoria histórica de los judíos y sus raíces. Convirtiendo en objetivo militar no solo a los mismos judíos y a sus objetos artísticos, religiosos y valiosos, sino que además intentó erradicar su patrimonio inmaterial, prohibiendo sus formas de transmisión cultural como su oralidad, creencias, costumbres y en general la memoria significativa para esta nación.

\section{Conclusiones}

En definitiva, la trasmisión oral de conocimientos, costumbres y tradiciones, al igual que los objetos con valor significativo; conforman el patrimonio cultural y permiten construir la identidad de una determinada comunidad o nación. Por ende, "el robo, el saqueo y el tráfico ilícito de objetos culturales son una negación pura y simple de los pueblos, reducen la historia a la categoría de mercancía. Además, causan daños graves, a menudo irreversibles, a la memoria colectiva, la cohesión social, al enriquecimiento mutuo" (UNESCO, 2014, p.2). Por ende, la protección de patrimonio cultural es un delito de guerra considerado como una cuestión de seguridad internacional (INTERPOL, s.f.b.).

El expolio y destrucción de patrimonio cultural se puede considerar como una amenaza a la construcción de la identidad de las naciones y, además, como un crimen de guerra, (UNESCO, citada en Torrecuadrada, 2013). Por lo tanto, se puede deducir que la finalidad de la destrucción y del expolio de bienes culturales por parte de Alemania en la segunda guerra mundial y de la gran mayoría de las invasiones hechas por los grandes países "no es un mero daño colateral, [o búsqueda de intereses materiales] sino que la 
intención es erradicar la presencia histórica y las vidas de la comunidad atacada" (Stanley, citado en Lizaranzu-Perinat, 2016, p.4). En tal caso, se puede tomar el expolio de patrimonio cultural como un arma de gran eficacia psicológica para aniquilar no sólo la memoria de los pueblos, su historia y sus tradiciones orales, sino que se trata del denominado genocidio cultural o etnocidio, por parte de paises hegemónicos que desconocen procesos culturales simbólicos permeados de oralidad en los bienes materiales tangibles e intangibles.

En efecto, en los conflictos armados se han presentado saqueos y búsquedas de los bienes materiales, no sólo para la comercialización clandestina de los mismos, sino que también se procede a borrar la memoria cultural, generando la desaparición de los pueblos, a tal punto de llegar a creer, por la falta de documentación y por la falta de transmisión oral, que nunca existió el genocidio, el saqueo, el expolio y con ello se procede a imponer otros aspectos ideológicos, otros referentes culturales los cuales niegan las tradiciones de los pueblos y comunidades, negando toda identidad cultural o religiosa que fuera diferente, demostrando de esta manera, su profunda devoción a la dominación sobre el otro.

\section{Recomendaciones}

Una vez concluida la investigación, se considera pertinente continuar la línea de investigación basada en las siguientes propuestas:

- Realizar un estudio enfocado a los vacíos de los acuerdos y de las organizaciones internacionales en la protección del patrimonio cultural.

- Se exhorta a desarrollar el estudio no sólo enfocado en el expolio nazi sino también en otros casos como lo fue la época colonial en Latinoamérica y la época actual con los conflictos armados en estos países.

En últimas, se necesita que más instituciones no gubernamentales, exentas de los poderes hegemónicos, revisen, fiscalicen, controlen y denuncien, las acciones en contra del patrimonio cultural tangible e intangible, por efectos de los conflictos. De esta manera, solo se recupera y preserva el acervo de conocimientos y técnicas que se transmiten de generación en generación. El valor social y cultural de esta transmisión de conocimientos de los grupos sociales minoritarios y mayoritarios de un Estado. 


\section{Referencias}

Albert, M. J. (2015, junio 6). El expolio de la guerra siria llega a Córdoba. El país. https:// elpais.com/cultura/2015/06/06/actualidad/1433621790_643116.html

Cagliani, M. (2015, octubre 18). ¿Qué es el método cualitativo?. Tendenzias.com. https:// tendenzias.com/ciencia/que-es-el-metodo-cualitativo/

Cardona, P. (2013, diciembre 29). La misión de los Monuments Men. Historias de la Segunda Guerra Mundial. https://www.historiassegundaguerramundial.com/operaciones/ los-monuments-men/?lang=en

CISION. (2019, marzo 11). Le Rapport Annuel Artprice du Marché de l'Art mondial 2018 : record du nombre d'adjudications depuis 1945, l'exigence des acheteurs en hausse. CISION PR Newswire. https:// www.prnewswire.com/news-releases/ le-rapport-annuel-artprice-du-marche-del-art-mondial-2018-record-du-nombre-dadjudications-depuis-1945-1-exigence-desacheteurs-en-hausse-849914139.html

Comunidad Andina [CAN]. (s.f.). Protección del patrimonio cultural. Recuperado el 4 de octubre de 2019. http://www.comunidadandina. org/Seccion.aspx? $\mathrm{id}=186 \&$ tipo=

Comunidad Andina [CAN]. (s.f.). Somos Comunidad Andina. Recuperado el 4 de octubre de 2019. http://www.comunidadandina.org/ Seccion.aspx? $\mathrm{id}=189 \&$ tipo $=\mathrm{QU} \&$ title $=-$-so$\underline{\text { mos-comunidad-andina }}$

Dinero. (2019, mayo 18). Así es como el arte se está volviendo un gran negocio en Colombia. Dinero. http://pruebas.dinero.com/Item/ ArticleAsync/269804? nextId=270063\&nex$\underline{\mathrm{tId}=270112}$
Domènech, N. (2009, octubre 14). España no salda cuentas con el expolio nazi. La Memoria Viva. https://lamemoriaviva.wordpress. com/2009/10/14/

El País. (2010, mayo 22). El robo de arte, una actividad especializada y nada romántica. El País. https://cincodias.elpais. com/cincodias/2010/05/22/sentidos/1274495236_850215.html

Espinosa-Sosa, M. E., Espinosa-Sosa, C. de las M. y Abad-Saínz, M. (2018). La oralidad, patrimonio inmaterial del campesino en la zona de Cartagena. Revista Caribeña de Ciencias Sociales. https://www.eumed.net/rev/ caribe/2018/10/oralidad-patrimonio-campesino.html

Feliciano, H. (2007, diciembre 01). El monstruo nazi del lago Ness. El País. https:// elpais.com/diario/2007/12/02/cultura/1196550003_850215.html

Fundación ILAM. (s.f.). Patrimonio Cultural. Recuperado el 14 de septiembre de 2019. https://ilamdir.org/patrimonio/cultural

Geertz, C. (1997). La interpretación de las culturas. Gedisa.

Gobierno de México. (s.f.). ¿Qué es la UNESCO? Recuperado el 4 de septiembre de 2019. https://mision.sre.gob.mx/unesco/index.php/ que-es-la-unesco

ICOM. (s.f.). Misiones y objetivos. Recuperado el 4 de octubre de 2019. https://icom.museum/ es/sobre-nosotros/misiones-y-objetivos/

INTERPOL. (s.f.). ¿Qué es INTERPOL? Recuperado el 04 de octubre de 2019. https://www. interpol.int/es/Quienes-somos/Que-es-INTERPOL 
INTERPOL. (s.f.). ¿Qué hacemos?. Recuperado el 04 de octubre de 2019. https://www.interpol. int/es/Delitos/Delitos-contra-el-patrimonio-cultural/Que-hacemos

INTERPOL. (s.f.). Bienes culturales en áreas de conflictos. Recuperado el 04 de octubre de 2019. https://www.interpol.int/es/Delitos/ Delitos-contra-el-patrimonio-cultural/Problematica-bienes-culturales

Lizaranzu-Perinat, M. T. (2016). El papel de la UNESCO en la protección del patrimonio en zonas de conflicto. Actualidad Jurídica Uría Menéndez, (24), 7-16. https://www.uria.com/ documentos/publicaciones/4960/documento/tribuna.pdf?id=6209

Manzini, L. (2009): Claves de lectura histórico - arquitectónica para la determinación del significado cultural de los bienes patrimoniales. El caso del patrimonio agroindustrial vitivinícola del Área Metropolitana de Mendoza [Tesis doctoral inédita]. Universidad de Mendoza.

Martorell-Linares, M. A. (2014). The monuments men. La fascinante aventura de los guerreros del arte que impidieron el expolio cultural nazi. Historia y politica: Ideas, procesos $y$ movimientos sociales, (31), 348-352. https:// dialnet.unirioja.es/servlet/articulo?codi$\mathrm{go}=4964886$

Ministerio de Cultura de Colombia. (2015, julio 30). Nuestro Patrimonio Cultural al alcance de todos. https://www.mincultura.gov.co/areas/ patrimonio/mes-del-patrimonio/patrimonio-cultural-al-alcance-de-todos/Paginas/ Nuestro-Patrimonio-Cultural-al-alcance-de-todos.aspx

Molano, O. L. (2007). Identidad cultural un concepto que evoluciona. OPERA, 7(7), 69-84. https://revistas.uexternado.edu.co/index. php/opera/article/view/1187
Monterey Institute for Technology and Education. (2011). Razonamiento Deductivo. http:// www.montereyinstitute.org/courses/Algebra1/U12L1T4_RESOURCE/topicText_ es.html

Monuments Men Foundation for the Preservation of Art. (s.f.). About us. Recuperado el 4 de octubre de 2019. https://www.monumentsmenfoundation.org/about

Mora, J. E. (2018, septiembre 18). El fascinante y oscuro mundo del robo de arte. Semanario Universidad. https://semanariouniversidad. com/cultura/el-fascinante-y-oscuro-mundo-del-robo-de-arte/

Paz, A., Cedeño, H. y Macías, A. (2016). Una aproximación sobre la construcción e investigación del patrimonio cultural. Dominio de las Ciencias, 2(4), 124-136. https://dialnet. unirioja.es/servlet/articulo? codigo $=5761630$

Piarpusan Pismac, E. D., Rosero Sosa, M. M., Solarte Suárez, A., Burbano Mora, A. A., Paz Feliciano, J. E., Jurado Agreda, M. D., b, J. C., Daza Alvarado, D. A. y Corella López, C. A. (2020). Nariño territorio intercultural. Etnoeducación, cultura e identidad de los pueblos de Nariño. Universidad Nacional Abierta y a Distancia. https://hemeroteca.unad.edu.co/ index.php/book/article/view/3967

Pinassi, C. A. (2018). Conflictos en torno al patrimonio cultural de Ingeniero White (Bahía Blanca, Argentina). Cuadernos De antropología Social, (48). https://doi.org/10.34096/cas. $\underline{\mathrm{i} 48.4100}$

Portafolio. (2020, agosto 04). Valor del oro cerró en máximo histórico este martes. El País. https://www.portafolio.co/internacional/ oro-cierra-en-maximos-historico-precio-deloro-hoy-4-agosto-2020-543353 
Ramos, C. M. (2003, abril 20). Las ONG que apoyan el arte. La Nación. https://www.lanacion.com.ar/lifestyle/las-ong-que-apoyan-elarte-nid489012/

Semana. (2013, abril 6). El tesoro que robaron los nazi en la Segunda Guerra Mundial. Revista Semana. https://www.semana.com/ cultura/articulo/el-tesoro-robaron-nazi-segunda-guerra-mundial/339051-3/

Semana. (2019, abril 13). ¿Europa le robó el arte a sus colonias?. Revista Semana. https://www. semana.com/cultura/articulo/europa-le-robo-el-arte-a-sus-colonias/609189/

Socorro, F., Reyes, G. y Trujillo, R. (2019). Escenarios irreales no utópicos. Revista Espacios, 40(3). http://www.revistaespacios.com/ a19v40n03/19400312.html

Torrecuadrada, S. (2013). Bienes incautados en tiempos de guerra: su protección y restitución. Biblioteca Nueva Fundación Ortega y Gasset.

UNAM. (s.f.). Axiología. Recuperado el 05 de octubre de 2019. https://programas.cuaed. unam.mx/repositorio/moodle/pluginfile. $\mathrm{php} / 1151 / \mathrm{mod} \_r e s o u r c e / c o n t e n t / 1 /$ contenido/index.html

UNESCO (2017, marzo 24). El Consejo de Seguridad de la ONU adopta una resolución histórica para la protección del patrimonio cultural. https://es.unesco.org/news/consejo-seguridad-onu-adopta-resolucion-historica-proteccion-del-patrimonio-cultural

UNESCO. (1972). Convención sobre la protección del patrimonio mundial, cultural y natural. UNESCO. http://whc.unesco.org/ archive/convention-es.pdf
UNESCO. (2014, marzo 10). UNESCO expresa preocupación por robo de obras de arte en Cuba y Guatemala. UNESCO Servicio de Prensa. http://www.unesco.org/new/es/media-services/single-view/news/unesco_expresses_its_concern_for_the_theft_of_works_of_art_i/

UNESCO. (s.f.). Patromonio cultural.https:// es.unesco.org/fieldoffice/santiago/cultura/ patrimonio

UNESCO. (s.f.a). Protección de los bienes culturales en caso de conflicto armado. https://wayback. archive-it.org/10611/20151106010027/ http://www.unesco.org/new/es/culture/themes/armed-conflict-and-heritage/

UNESCO. (s.f.b). Actividades y logros de la UNESCO en la protección del Patrimonio Cultural Subacuático. http://www.unesco.org/new/es/ culture/themes/underwater-cultural-heritage/protection/unescos-work/

UNESCO. (s.f.c). El Convenio de UNIDROIT de 1995. http://www.unesco.org/new/es/culture/themes/illicit-trafficking-of-cultural-property/1995-unidroit-convention/

UNIDROIT. (2014, junio 09). Presentacion convenio de UNIDROIT sobre los bienes culturales robados o exportados ilícitamente (Roma, 1995). https://www.unidroit.org/overviecp/spanish

Verón, E. (2018). La protección del patrimonio cultural contra el tráfico ilícito de bienes culturales. Dykinson.

Visón, I. (2003). Editorial: frente a la historia, los museos y el patrimonio en los períodos de conflicto y post-conflicto. Museum INTERNATIONAL, 55(3-4), 4-8. https://unesdoc. unesco.org/ark:/48223/pf0000133506_spa 